\title{
The impact of an electron and hole trapping coefficient on nonlinear phenomena in photorefractive multiple quantum well structures
}

\author{
Błażej Jabłoński, Andrzej Ziółkowski, Agnieszka Branecka, Ewa Weinert-Rączka \\ West Pomeranian University of Technology in Szczecin, Piastów 17, 70-310 Szczecin
}

Received December 07, 2016; accepted December 11, 2016; published December 31, 2016

\begin{abstract}
Semiconductor photorefractive quantum wells belong to materials with strong optical nonlinearity. One of the parameters that may affect the course of nonlinear phenomena in these materials is the electron and hole trapping coefficient. We present the results of a numerical analysis aimed to find out how electric field-dependen trapping coefficients affect the process of space-charge field formation in multiple quantum wells in the phenomenon of photorefractive twowave mixing.
\end{abstract}

Photorefractive multiple quantum wells are characterized by high sensitivity and relatively short response time [1-2]. A characteristic feature of these materials is the occurrence of bipolar transport. This reduces the impact of diffusion on the phenomenon and necessitates the application of an external electric field. For GaAs/AlGaAs structures working in Franz-Keldysh geometry (electric field applied parallel to quantum wells), it is observed that electron mobility depends on field intensity. This phenomenon affects the course of two-wave mixing, causing the shift of a space-charge field relative to the interference pattern and enabling oneway exchange of energy between the waves [1-3].

Another parameter that may have an impact on the course of nonlinear phenomena is the electron and hole trapping coefficient. The study [5] presents the influence of a strong electric field on the values of cross-sections for recombination to traps for bulk GaAs with EL2 defects. On the other hand, in studies of photorefractive two-wave mixing in GaAs/AlGaAs quantum wells the authors adopt a fixed value of these coefficients [1-4]. This approach is justified in the case of a small contrast of the interference pattern. Since experimental works often involve systems with deep modulation of light intensity, in which there are large local changes of electric field intensity, it is worth examining what the influence of the field dependence of carrier trapping coefficients is. In this work, we present numerical studies of the impact of this relation on the process of space-charge field formation under the stationary interference pattern.

The photorefraction phenomenon in semi-insulating materials can be described with the band transport model PDDT (photogeneration - diffusion - drift - trapping) [2]. In descriptions of semiconductor structures the occurrence of one level of deep traps and one level of shallow dopants in the energy gap is usually assumed.
Here we scrutinize the material in which shallow dopants are acceptors with concentration $N_{A}$, whereas donors with concentration $N_{D}$ play the role of deep trapping levels. The relationship between the concentrations of donors and acceptors is described by the compensation coefficient $r=N_{A} / N_{D}$. In addition, we assume the total ionization of shallow acceptors, i.e. $N_{A}^{-}=N_{A}=$ constans. They do not take part in photorefractive transitions, but have an important impact on the initial concentration of ionized donors. Ionized donors with the concentration $N_{D}^{+}$act as trap centers for electrons, while non-ionized donors with the concentration $N_{D}^{0}=N_{D}-$ $N_{D}^{+}$are hole trap centers. The photorefraction in the structures of multiple quantum wells in the Franz-Keldysh geometry can be described in the same way as for bulk materials. For negligible lateral transport, i.e. for the carriers trapped in quantum wells, we can use the onedimensional model, based on the equation system describing the generation, transport and recombination of electrons and holes [2]:

$$
\begin{aligned}
& \frac{\partial n_{e}}{\partial t}=\left(\alpha+S_{e} N_{D}^{0}\right) \frac{I}{h v}+\beta_{e} N_{D}^{0}-\gamma_{e}(E) n_{e} N_{D}^{+}+\frac{1}{q} \frac{\partial j_{e}}{\partial x} \\
& \frac{\partial n_{h}}{\partial t}=\left(\alpha+s_{h} N_{D}^{+}\right) \frac{I}{h v}+\beta_{h} N_{D}^{+}-\gamma_{h}(E) n_{h} N_{D}^{0}-\frac{1}{q} \frac{\partial j_{h}}{\partial x} \\
& \frac{\partial \rho}{\partial t}=-\frac{\partial}{\partial x}\left(j_{e}+j_{h}\right), \\
& j_{e}=q \mu_{e}(E) E n_{e}+k_{B} \frac{\partial}{\partial x}\left(\mu_{e}(E) T_{e}(E) n_{e},\right. \\
& j_{h}=q \mu_{h} E n_{h}-k_{B} \mu_{h} T_{h} \frac{\partial n_{h}}{\partial x} \\
& \frac{\partial E}{\partial x}=\frac{\rho}{\varepsilon_{0} \varepsilon} \\
& \rho=q\left(N_{D}^{+}-N_{A}^{-}+n_{h}-n_{e}\right)
\end{aligned}
$$

where the indices $e$ and $h$ refer to electrons and holes; $n$ describes the concentration of carriers; $j$ is the current density, $\beta$ is the thermal generation rate, $E$ denotes the total electric field intensity, and $\gamma(E)$ is the fielddependent trapping coefficient, $\alpha$ describes the interband absorption. The coefficients $s_{e}$ and $s_{h}$ specify the crosssections for photoionization of traps, $\mu$ represents the mobility of carriers, whereas the quantities $T_{e}$ and $T_{h}$ describe the temperature of electrons and holes, $q$ is the elementary electrical charge, $k_{B}$ is Boltzmann's constant, $\varepsilon$ is the dielectric constant, and $\varepsilon_{0}$ is the vacuum permeability. 
The dependence of electron mobility on electric field $\mu_{e}(E)$, present in Eqs. (1), is related to the fieldinduced increase of lectron energy and resulting occupation of the side valleys of a conduction band, in which mobility is lower - the so called "hot electrons" effect. In a simple two-valley model [6] electron mobility is presented as a weighted average:

$\mu_{e}(E)=\mu_{\Gamma} f(E)+\mu_{L}[1-f(E)]$,

where $\mu_{\Gamma}, \mu_{L}$ define the electron mobility in the central and side valleys, while $f(E)$ is a distribution function expressing relative occupation of the central valley [7].

The trapping coefficient value can be determined by the equation $\gamma_{i}=\sigma_{(i)} v_{t h(i)}$, where value $\sigma_{(i)}$ defines the cross section for recombination to traps, and $v_{t h}$ is the average thermal velocity of carriers, $i=e, h$, for electrons and holes, respectively. In previous publications describing photorefractive phenomena occurring within SI-MQW structures [1-4] the value of a cross-section for recombination to traps of both electrons and holes is assumed to be constant.

In the case of two waves interference, light intensity has the form:

$$
I(x, t)=I_{0}(t)[1+m \cos (K x)],
$$

where $I_{0}(t)$ describes the time dependence of average light intensity, $K$ is the grating constant, $m=$ $2\left(I_{1} I_{2}\right)^{1 / 2} / I_{0}$ defines the modulation depth of interference pattern. In the case of a small modulation depth, $m \leq$ 0.1 , when the minimum and maximum values of electric field differ slightly, the use of constant trapping coefficients is justified. However, in the case of high modulation depth, when these differences are significant, we should examine the impact of local changes of electric field on the trapping coefficients and, as a result, on all processes described by Eqs. (1). An example of electric field distributions for various modulation depths is presented in Fig. 1.

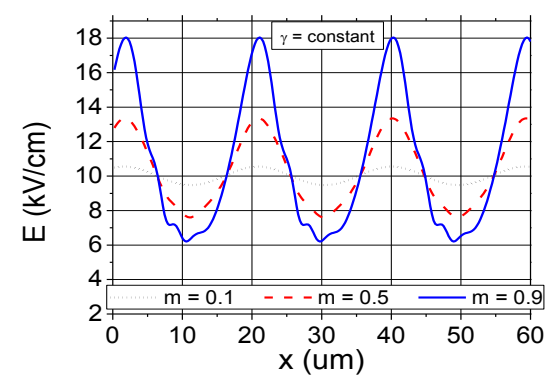

Fig.1. Spatial distribution of the electric field in the SI-MQW structure for $\gamma=$ constant, external electric field $E_{A}=10 \mathrm{kV} / \mathrm{cm}$ and various modulation depths. Parameters used in the simulation are listed in Tab.1.

The material parameters of the structure [2, 4] and the parameters used in the calculations are given in Table 1.
Table 1: The material parameters of the structure and the parameters used in calculations.

\begin{tabular}{lll}
$\begin{array}{l}\text { Cross section for } \\
\text { photoionization of traps }\end{array}$ & $s_{e}=1 \cdot 10^{-17} \mathrm{~cm}^{2}$ & $s_{h}=1 \cdot 10^{-16} \mathrm{~cm}^{2}$ \\
$\begin{array}{l}\text { Cross section for } \\
\text { recombination to traps }\end{array}$ & $\sigma_{e}=1 \cdot 10^{-14} \mathrm{~cm}^{2}$ & $\sigma_{h}=1 \cdot 10^{-14} \mathrm{~cm}^{2}$ \\
Carrier mobility & $\mu_{e}=5000 \mathrm{~cm}^{2} / V s$ & $\mu_{h}=300 \mathrm{~cm}^{2} / V s$ \\
$\begin{array}{l}\text { Thermal generation rate } \\
\text { Interband absorption }\end{array}$ & $\beta_{e}=0,5 \mathrm{~s}^{-1}$ & $\beta_{h}=1 \mathrm{~s}^{-1}$ \\
coefficient & $\alpha=10^{4} \mathrm{~cm}^{-1}$ & \\
Average refractive index & $n_{f}=3,55$ & \\
$\begin{array}{l}\text { Donor concentration } \\
\text { Donor concentration }\end{array}$ & $N_{D}=5 \cdot 10^{16} \mathrm{~cm}^{-3}$ & \\
ratio & $r=0.8$ & \\
Grating period & $\Lambda=20 \cdot 10^{-9} \mathrm{~m}$ & \\
Writing wavelength & $\lambda=830 \cdot 10^{-9} \mathrm{~m}$ & \\
Average light intensity & $I_{0}=10 \mathrm{~mW} / \mathrm{cm}^{2}$ \\
Modulation depth & $m=0.9$ \\
Dielectric constant & $\varepsilon=12.58$ & \\
\hline
\end{tabular}

The influence of an electric field on the values of crosssections for recombination of electrons and holes to traps for bulk GaAs, examined by capacitance spectroscopy and described in Ref. [5], is presented in Fig. 2.

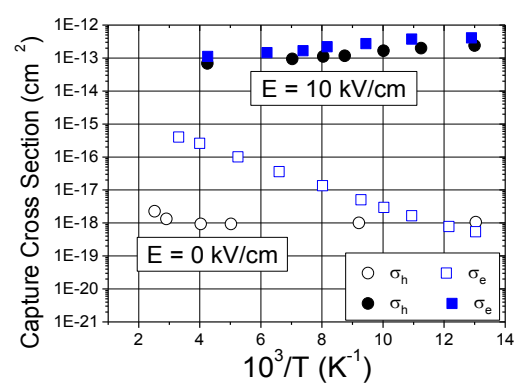

Fig. 2. The values of the cross-sections for recombination of electrons $\sigma_{e}$ and holes $\sigma_{h}$ to traps for $E=0 \mathrm{kV} / \mathrm{cm}$ and in the presence of a strong electric field $E=10 \mathrm{kV} / \mathrm{cm}$ for GaAs with EL2 defects [5].

The values of trapping coefficients for the same thermal velocities as in [4], namely $v_{t h(e)}=4.5 \cdot 10^{7} \mathrm{~cm} / \mathrm{s}$ and $v_{t h(h)}=1.8 \cdot 10^{7} \mathrm{~cm} / \mathrm{s}$, are shown in Table 2 .

Table 2: The values of trapping coefficients

\begin{tabular}{l|cc}
\hline \multirow{4}{*}{ Values derived on } & $E=0 \mathrm{kV} / \mathrm{cm}$ & $E=10 \mathrm{kV} / \mathrm{cm}$ \\
\cline { 2 - 3 } the basis of [5] & $\sigma_{e}=4 \cdot 10^{-16} \mathrm{~cm}^{2}$ & $\sigma_{e}=1 \cdot 10^{-14} \mathrm{~cm}^{2}$ \\
& $\sigma_{h}=1 \cdot 10^{-18} \mathrm{~cm}^{2}$ & $\sigma_{h}=2 \cdot 10^{-14} \mathrm{~cm}^{2}$ \\
& $\gamma_{e}=2.0 \cdot 10^{-8} \mathrm{~cm}^{3} / \mathrm{s}$ & $\gamma_{e}=4.5 \cdot 10^{-7} \mathrm{~cm}^{3} / \mathrm{s}$ \\
& $\gamma_{h}=2.0 \cdot 10^{-11} \mathrm{~cm}^{3} / \mathrm{s}$ & $\gamma_{h}=3.6 \cdot 10^{-7} \mathrm{~cm}^{3} / \mathrm{s}$ \\
\hline Parameters used & $\sigma_{e, h}=1 \cdot 10^{-14} \mathrm{~cm}^{2}$ & $\gamma_{e}=4.5 \cdot 10^{-7} \mathrm{~cm}^{3} / \mathrm{s}$ \\
in the study [4] & $\gamma_{h}=1.8 \cdot 10^{-7} \mathrm{~cm}^{3} / \mathrm{s}$ \\
\hline
\end{tabular}

In order to examine the impact of the dependence of electron and hole trapping coefficients on the electric field, we assumed that the functions $\gamma(E)$ can be described by the relations:

$\gamma_{i}(E)=\gamma_{i_{0}}+\gamma_{i_{\max }} \cdot\left(1-e^{-\alpha_{i} \cdot E}\right)$ 
where $\gamma_{i_{0}}$ are the values of the trapping coefficient for $E=0$. The relations $\gamma_{e}(E)$ and $\gamma_{h}(E)$, matched to the experimental results presented in [5], are shown in Fig. 3.
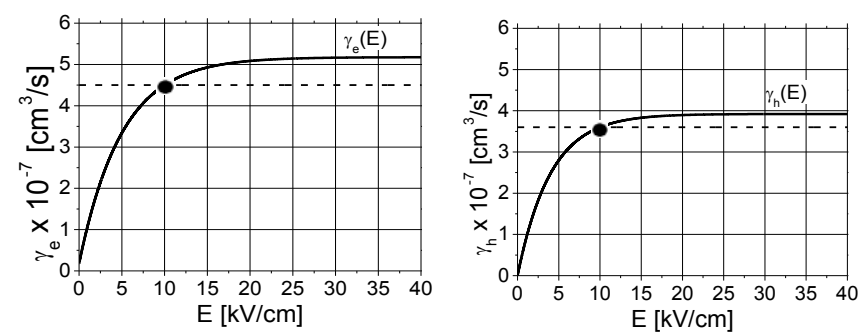

Fig. 3. The dependence of the trapping coefficient for electrons (a) and holes (b) on the electric field obtained from Eq. (4) with parameters matched to the results described in [5], $\left(\gamma_{e_{\max }}=5.0 \cdot 10^{-13}, \gamma_{h_{\max }}=\right.$

$$
\left.3.9 \cdot 10^{-13}, \alpha_{e}=2.0 \cdot 10^{-6} \mathrm{~cm} / \mathrm{kV}, \alpha_{h}=2.5 \cdot 10^{-6} \mathrm{~cm} / \mathrm{kV}\right) .
$$

The assumed $\gamma(E)$ relations include quasi-linear changes within small and medium field ranges, the value compatible with [5] for $10 \mathrm{kV} / \mathrm{cm}$ field and the saturation for a higher field. The spatial distributions of the total electric field in the structure, derived from the equations (1) for various values of an external electric field are shown in Fig. 4.
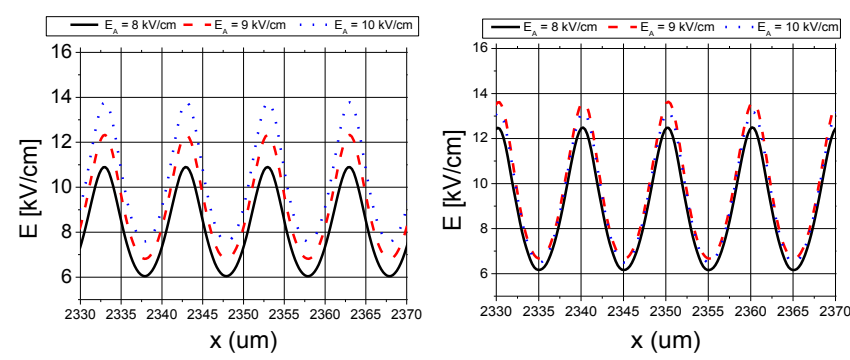

Fig. 4. Spatial distribution of an electric field in the steady state for different values of external electric field applied to the structure, for the constant (a) and dependent on electric field trapping coefficients (b).

Figure 5 shows the process of space-charge field formation for different trapping coefficients. The solid line represents the evolution of the total electric field in the structure for varying $\gamma(E)$ and the dashed line for a constant one. As seen, the space-charge field formation time in the model taking into account the variation of parameter $\gamma(E)$ is longer.

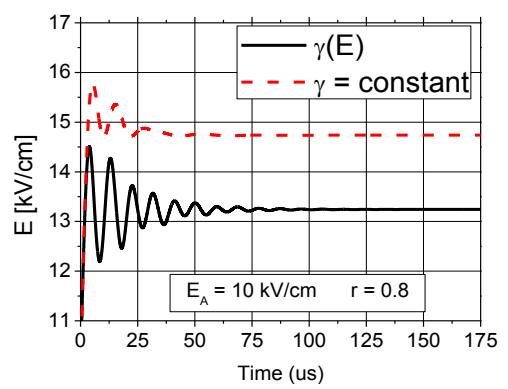

Fig. 5. Evolution of total electric field amplitude (space-charge field + field applied to the structure) for constant and variable values of coefficient $\gamma$.
In conclusion, this paper presents the results of numerical calculations concerning the impact of the electric field-dependent electron and hole trapping coefficients on the phenomenon of space-charge field formation in the SI-MQW structure in the presence of a steady interference pattern. For examined typical structures, the dependence of coefficients $\gamma_{e}$ and $\gamma_{h}$ on the electric field only slightly affects its spatial distributions, but it permits to obtain higher amplitude of the spacecharge electric field at lower intensity of the external field. In addition, the presented model may explain the longer duration of the space-charge field formation process than expected in the model with constant coefficients (Fig. 5).

\section{References}

[1] Q. Wang, R.M. Brubaker, D.D. Nolte, M. R. Melloch, J. Opt. Soc. Am. B 9, 1626 (1992).

[2] D.D. Nolte, M.R. Melloch, in Photorefractive effects and Materials ed. by D. D. Nolte (Kluwer Academic, Boston, 1995).

[3] D.D. Nolte, J. Appl. Phys. 85, 6259 (1996).

[4] Q. Wang, R.M. Brubaker, D.D. Nolte, J. Opt. Soc. Am. B 9, 1773 (1994).

[5] V. Ya. Prinz, S.N. Rechkunov, Phys. Stat. Sol. (B) 118, 159 (1983).

[6] S.M. Sze, Physics of Semiconductors Devices (Wiley, New York 1981).

[7] B. Jabłoński, JNOPM 23, 1450029 (2014). 